\title{
DEVELOPMENT OF ARTIFICIAL LATERAL-LINE FLOW SENSORS
}

\author{
Zhifang Fan, Jack.Chen, Jun Zou, Junjun Li, Chang Liu and Fred Delcomyn* \\ Micro Actuators, Sensors and Systems Group \\ Micro and Nanotechnology Laboratory \\ * Department of Entomology \\ University of Illinois at Urbana-Champaign, Urbana, IL 61801
}

\begin{abstract}
SUMMARY
We report the development of an underwater flow sensor that is based on biological inspiration - the biological lateral line sensors. The design, fabrication process and initial testing results for an artificial lateral line sensor are reported. The fabrication is based on a highly efficiently three-dimensional assembly process, used in conjunction with silicon micromachining techniques.
\end{abstract}

\section{BACKGROUND}

Evolved over hundreds of millions of years, many fish species rely on lateral line sensors to monitor surrounding flow fields for maneuvering and survival under water [1]. It is conjectured that fish is equipped with distance touch sense of underwater obstacles, predators, and prey. A lateral line system, shown in Fig. 1, consists of an array of distributed sensor nodes (so called neuromasts) that span the length of the fish body. Each sensor node, in turn, consists of a cluster of hair cells embedded in protective, gel-like domes. In certain fish species, the lateral line sensor nodes may be directly exposed at the surface of the skin. In others, the sensor nodes may be hidden in subdermal channels in order to minimize wear and damage. An individual biological hair cell is called a mechanoreceptor. It consists of a vertical cilium attached to a neuron. The fundamental principle of a single haircell is as follows. When the cilium of the haircell is bent by the local fluid flow, the neuron attached to the cilium stretches and produces action potentials.

An array of haircells with different length and hence frequency characteristics reside at each sensor node. This slows fish to extend the dynamic range and frequency spectrum of the environmental awareness.

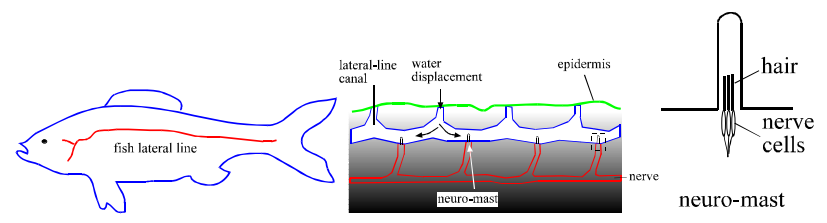

Figure 1: Schematic diagram of fish lateral line sensor system. The lateral line is distributed through the fish body surface. A lateral line consists of many neuronal nodes, called neuromasts. Each neuromast consists of an array of haircells embedded in a protective dome.

Engineered flow sensors have been developed in the past based on a number of sensing principles, including thermal (hot-wire) anemometry [2], Doppler frequency shift, and indirect inference from pressure differences [3-4]. Hot-wire anemometers use fine heating elements that double as temperature sensors. The local flow rate is inferred from the extent of forced convective heat transfer from the hot wire. The majority of existing hotwire anemometers are manufactured using macroscopic machining methods. Micromachined hot-wire anemometers have also been developed in recent years [5]. Sensors based on Doppler frequency shifts consist of an acoustic launcher and a receiver. The speed of fluid in between the launcher and the receiver is interpreted using Doppler frequency shift. The overall size of the device is generally large. Existing flow sensors based on pressure distribution measurements also have large sizes and are generally not suitable for forming distributed arrays.

In the past two decades, several research groups have developed micromachined flow sensors that are based on a variety of sensing principles including the three principles mentioned above [6-13]. Microfabrication offers the benefits of high spatial resolution, fast time response, integrated signal processing, and potentially low costs. In addition to flow sensors, boundary-layer shear stress sensors have been realized using floating element methods [14] and thermal transfer principles [15]. However, shear stress sensors are located directly on the fluid-solid boundary and do not provide adequate information about mean stream flow velocity.

This work offers an alternative method for underwater flow sensing. The sensor is uniquely based on biological inspiration. Each sensor covers small footprint and therefore high density integration is possible. The vertical cilium extends into the boundary layer flow instead of residing at the non-slip interface, allowing greater flow sensitivity.

\section{SENSING PRINCIPLE}

The schematic diagram of the artificial lateral line sensor is shown in Fig. 2. Each sensor consists of an in-plane fixed-free cantilever with a vertical artificial cilium attached at the distal, free end. External flow parallel to the sensor substrate imparts upon the vertical cilium. Due to rigid connection between the in-plane cantilever and the vertical cilium, a mechanical bending moment is transferred to the horizontal cantilever beam, inducing strain at the base of the cantilever beam. The magnitude of the induced strain can be sensed by many means, for example by using integrated piezoresistive sensors. The overall sensor system may use an array of individual sensory nodes with systematically varying positions, height of cilia, and orientation. Such a distributed sensor array can provide information about flow structure evolution and increase the accuracy of flow field interpretation.

In this paper, we focus on the development and characterization of individual hair-cell-like sensors. 
A comprehensive understanding of the relation between the output (relative change of resistance) and the mean flow velocity must take into consideration many possible flow conditions. For complex situations, it may be necessary to use the finite-element numerical method to estimate the sensor output precisely. A comprehensive treatment on the fluid mechanics aspect is beyond of the scope of this paper, which focuses on the discussion of new design and fabrication methods. Nonetheless, the relationship can be analytically expressed if the flow condition is simple and well defined [16].

As a first-order approximation, the magnitude of the output signal is proportional to $u_{0}^{2}$.

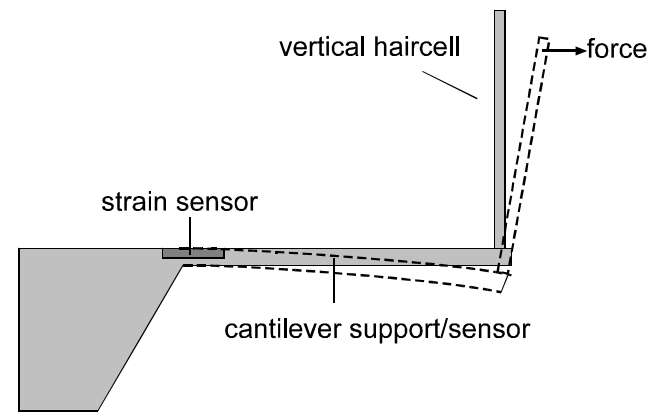

Figure 2: Schematic diagram of a single artificial haircell sensor consisting of a horizontal cantilever with a vertical cilium attached at the free end. We sense the bending of the vertical cilium using the strain sensor located at the base of the horizontal cantilever.

\section{FABRICATION PROCESS}

Flow sensors based on flow moment transfer have been reported in the past. The previously reported devices fall into two categories. A first type utilizes monolithic cantilever beams fabricated within the plane of the wafer by using bulk micromachining methods [6]. Such a sensor is not applicable for large array formation, as each sensor must be discretely packaged. A second type utilizes a vertical, high-aspect-ratio cilium that is connected to micromachined force-sensing components $[17,18]$. The overall yield and repeatability of the sensor are generally low because the fabrication process is not monolithic.

\section{The PDMA Three-Dimensional Assembly Process}

In this study, we leverage a recently developed, efficiently threedimensional assembly process to realize the vertical cilium, a key component of the hair cell. The vertical cilium is realized using a three-dimensional assembly technique called plastic deformation magnetic assembly (PDMA) [19].

\section{Hair Cell Fabrication Process}

The overall fabrication process of the artificial hair cell is discussed in the following and illustrated in Fig. 3. The process begins with deposition of a silicon dioxide layer by thermal oxidation. The oxide layer is patterned and selectively removed to provide openings for forming piezoresistive sensors by the diffusion doping process. Boron pre-deposition is performed at $950^{\circ} \mathrm{C}$ for $15 \mathrm{~min}$. It is followed by oxidation drive-in at $1100^{\circ} \mathrm{C}$ for 2 hours, reaching an oxidation thickness of $500 \mathrm{~nm}$ (Fig. 3c).
The targeted sheet resistivity of the doped resistor is $100 \Omega / \square$. The gauge factor of such a doped piezoresistive sensor is a function of the doping concentration and the substrate orientation [20-21]. At such a doping level, the gauge factor is optimized. In addition, the relatively high sheet resistivity allows a relatively large resistance value (on the order of 2-5 $\mathrm{k} \Omega$ ) to be realized within a small device footprint.

The wafer is etched from the backside using wet anisotropic etching or deep reactive ion etching until a silicon membrane with desired thickness is achieved (Fig. 3e). Structures that are pertinent to the PDMA process are then deposited and patterned. We deposit a layer of thin-film copper (300 nm thick) and pattern it photolithographically. A 600-nm-thick gold thin film is deposited by using the lift-off technique. This ensures good step coverage over the boundary of the sacrificial material and minimizes stress concentration. The subsequently electroplated Permalloy material is $10 \mu \mathrm{m}$ (Fig. 3f) in thickness.

(a)

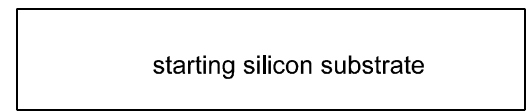

(b)

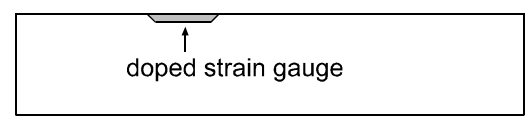

(c)

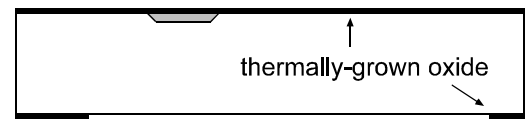

(d)

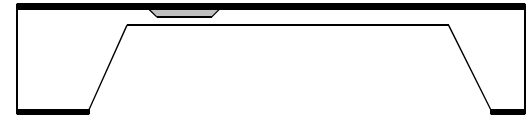

(e)

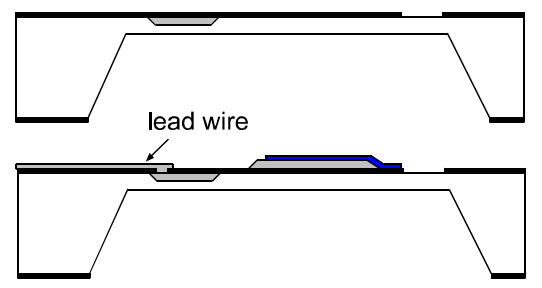

(g)
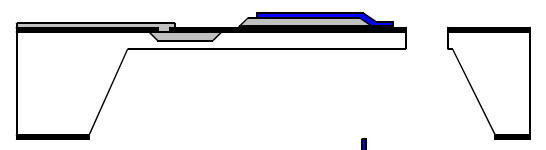

(h)

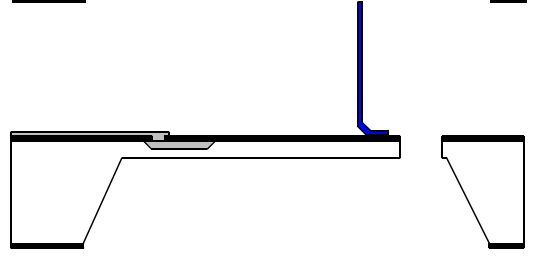

Figure 3: Fabrication process of an artificial lateral line sensor with integrated signal processing units. In the final step, the vertical cilium is assembled into three-dimensional position. It can be further protected and insulated by uniform, conformal deposition of Parylene thin film.

The wafer is then etched from the front side to define the cantilever beams (Fig. 3g). Following this step, the wafer is immersed in diluted $\mathrm{HCl}$ solutions to remove the copper 
sacrificial layer and to free the cantilever beam. The PDMA process is performed, followed by drying of the wafer (Fig. 3h). The SEM micrograph of a representative device is shown in Fig. 4. The length, width, and thickness of the in-plane cantilever are $1100 \mu \mathrm{m}, 180 \mu \mathrm{m}$, and $17 \mu \mathrm{m}$, respectively. The height, width and thickness of the vertical cilium are $820 \mu \mathrm{m}, 100 \mu \mathrm{m}$, and 10 $\mu \mathrm{m}$, respectively.

Improvement has been made to the original PDMA process to increase the yield of assembly. The cross-sectional view of the bending region according to the original PDMA assembly method introduces two problems. First, the beam has been prone to breaking during the assembly process due to excessive stress concentration at the step caused by the edge of the sacrificial layer. Secondly, the exact location of bending is uncertain, being between points A and B. This affects the uniformity of the angle of assembled hair-cell cilia.

A structural modification has been proposed and validated (Fig. 5). An extended patch made of $10-\mu \mathrm{m}$-thick electroplated Permalloy is electroplated to overlap with the edge of the sacrificial layer. This produces two noticeable improvements. First, the exact location of bending is much more easily controlled. Wse confirmed that the bending occurs at point $\mathrm{C}$. Secondly, bending will not involve the step-coverage region and therefore the stress-induced fracture is removed.

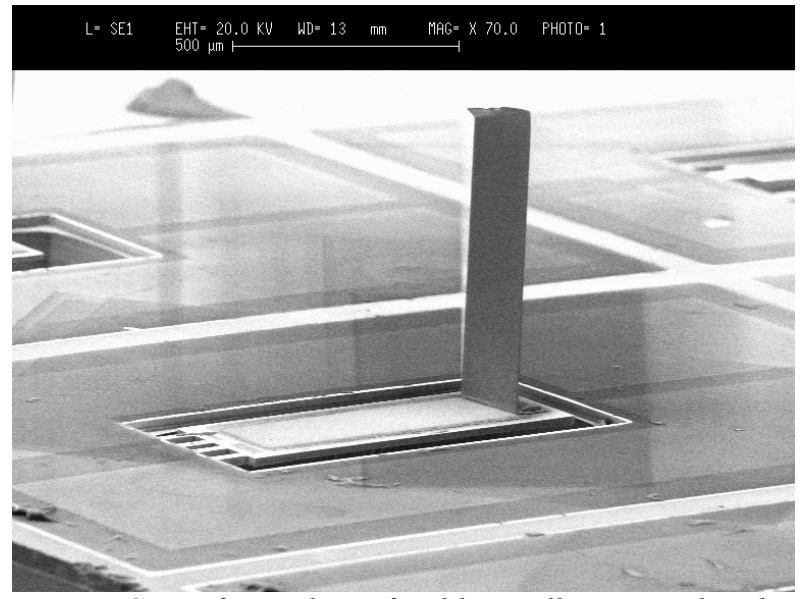

Figure 4: SEM of a single artificial hair cell sensor. The cilium is $820 \mu \mathrm{m}$ tall.

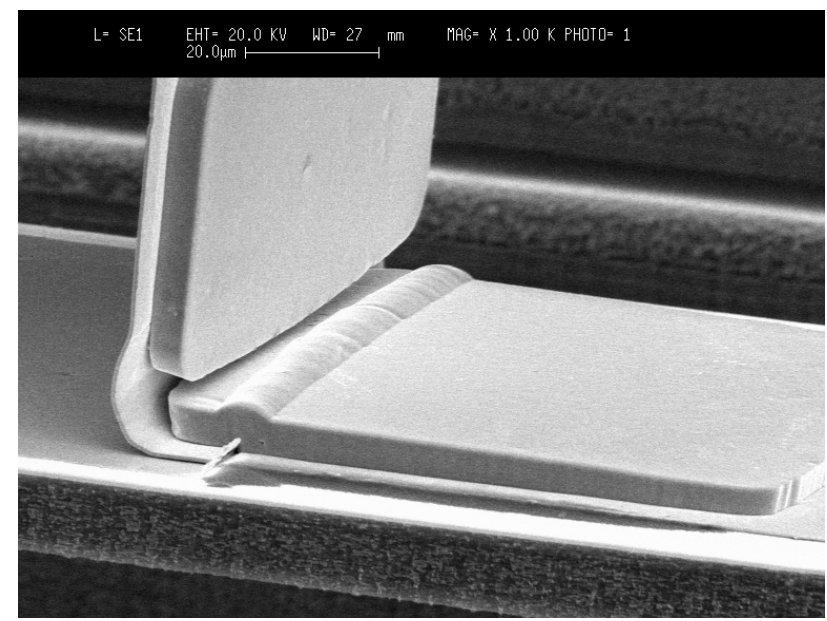

Figure 5: SEM of the plastically bent region at the base of the vertical cilium.
We have demonstrated that it is possible to strengthen the plastically deformed joint of the vertical cilium by using localized electroless plating methods. First, circuit elements and wire leads are protected from shorting by an organic insulator such as the photoresist. A thin layer of electrolessly-plated gold is grown onto the exposed vertical cilium at $95^{\circ} \mathrm{C}$ for $10 \mathrm{~min}$ with the growth rate being approximately $0.3 \mu \mathrm{m} / \mathrm{min}$.

The fabricated device is coated with 300-nm-thick uniform Parylene deposition for increasing strength and electrical insulation (Fig. 6). The insulating coating allows sensors to operate in conducting fluids. The deposited material, however, increases the force constant of the cantilever beam. The thickness must be optimized with respect to two competing concerns, electrochemical protection and sensitivity.

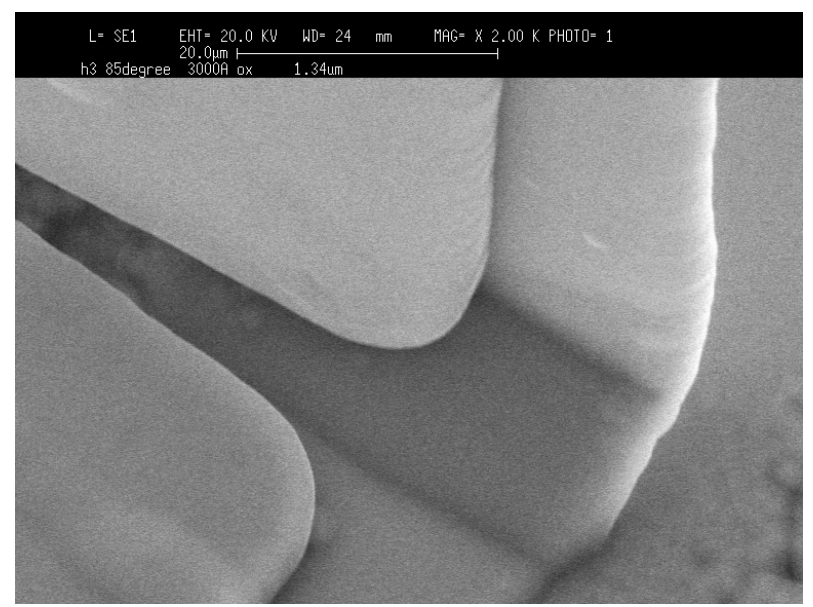

Figure 6: Enlarged view of the plastically bent region coated with Parylene thin film for strengthening and electrical insulation.

\section{RESULTS AND DISCUSSIONS}

The sensors are mounted on a thin glass plate, which is placed within a laminar flow water tunnel. One edge of the glass is polished to present a sharp profile facing the flow. The sensor is located $1 \mathrm{~mm}$ from the leading (sharp) edge. The flow rate of the water tunnel is varied manually. Laminar flow with $u_{0}$ ranging from 0 to $1 \mathrm{~m} / \mathrm{s}$ passes the sensor element. The flow impacts the cilium at its broad side, i.e., parallel to the long axis of the substrate cantilever. The sensor is biased under constant voltage $(1 \mathrm{~V})$ and the output current is monitored. The output response is shown in Fig. 7. The best-fit curve follows a second-order polynomial expression.

We are currently developing arrayed sensors to characterize the response of the sensors with respect to the orientation to the flow and to the height of the artificial cilium. It is believed that in order to increase the sensitivity, one can increase the height of the cilium or reduce the thickness of the in-plane cantilever. However, the vertical cilium should not be excessively tall as this may increase the chance of damage. For long cilia and higher flow rates, the interpretation of flow is more challenging as multiple flow regimes (laminar, transition, or turbulent) may be applied through the length of the cilium. To understand the exact nature and profile of local flow would require multiplesensor clusters much like the biological sensors. 


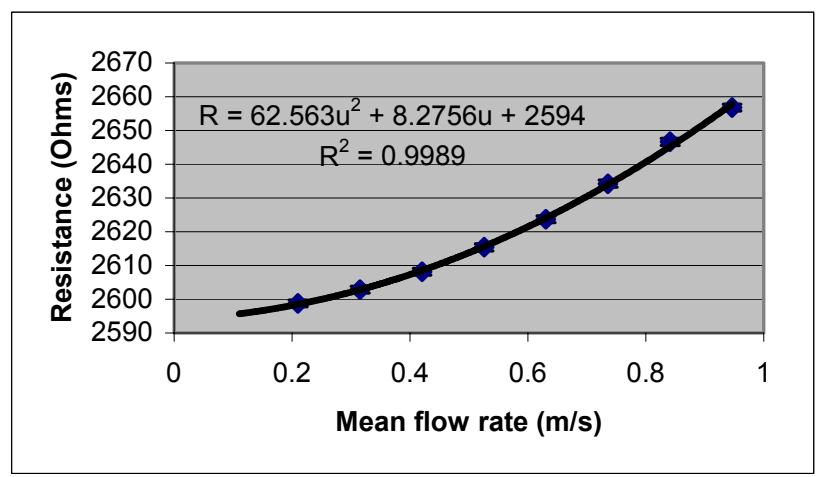

Figure 7: Measurement results showing output current vs. flow rate of a representative sensor under constant voltage biasing.

\section{CONCLUSIONS}

We have developed the design and fabrication process of an individual micromachined artificial lateral-line sensor for flow sensing applications. The sensor is realized using combined bulk micromachining methods and an efficient threedimensional assembly method. Sensors can be strengthened using electroless plating and Parylene conformal coating. They can be realized monolithically using combined bulk micromachining and three-dimensional assembly methods.

\section{ACKNOWLEDGMENTS}

The authors wish to thank the sponsors of this research work AFOSR BioInspired Concepts (BIC) Program, NSF Career Award and NASA.

\section{REFERENCES}

1. C.E. Bond, Biology of fishes, 2nd Ed., Saunders (1996).

2. A.E. Perry, Hot wire anemometry, Claredon Press (1982).

3. M. Richter, M. Wackerle, P. Woias, B. Hillerich, "A novel flow sensor with high time resolution based on differential pressure principle," Proc. $12^{\text {th }}$ Int. Conf. On Micro Electro Mechanical Systems, Orlando, FL, pp. 118-123 (1999).

4. O.K. Rediniotis, E. Johansen and T. Tsao, "MEMS-based probes for velocity and pressure measurements in unsteady and turbulent flowfields," Proc. 37th Aerospace Sciences Meeting, Reno, Nevada (1999).

5. F. Jiang, Y.C. Tai, C.M. Ho, K. Rainer, M. Garstenauer, "Theoretical and experimental studies of micromachined hot-wire anemometer," Digest IEEE Int. Electron Devices Meetings (IEDM), pp. 139-142, San Francisco (1994).

6. N. Svedin, E. Stemme, G. Stemme, "A static turbine flow meter with a micromachined silicon torque sensor," Technical Digest (MEMS 2001) 14th IEEE International Conference on Micro Electro Mechanical Systems, pp.20811, Interlaken, Switzerland (2001).

7. L. Lofdahl, E. Kalvesten, T. Hadzianagnostakis, G. Stemme, "An integrated silicon based wall pressure-shear stress sensor for measurements in turbulent flows," Proc.
1996 International Mechanical Engineering Congress and Exposition, pp. 245-51, New York, NY, USA (1996).

8. N. Svedin, E. Kalvesten, E. Stemme, G. Stemme, "A new silicon gas-flow sensor based on lift force," Journal of Microelectromechanical Systems, vol.7, no.3, pp.303-8, (1998).

9. T. Ebefors, E. Kalvesten, G. Stemme, "Three dimensional silicon triple-hot-wire anemometer based on polyimide joints," Proc. Eleventh Annual International Workshop on Micro Electro Mechanical Systems, pp.93-98 (1998).

10. E. Kalvesten, C. Vieider, L. Lofdahl, G. Stemme G, “An integrated pressure-flow sensor for correlation measurements in turbulent gas flows," Sensors \& Actuators A-Physical, Vol. A52, No.1-3, pp. 51-8 (1996).

11. P. Enoksson, G. Stemme, E. Stemme, "A Coriolis mass flow sensor structure in silicon," Proceedings. The Ninth Annual International Workshop on Micro Electro Mechanical Systems, Machines and Systems, pp.156-61 (1996).

12. L. Lofdahl, G. Stemme, B. Johansson, "Silicon based flow sensors used for mean velocity and turbulence measurements," Experiments in Fluids, Vol.12, No.4-5, pp.270-6 (1992).

13. G.T.A. Kovacs, Micromachined transducers sourcebook, McGraw-Hill (1998).

14. Padmanabhan, H. Goldberg, K.D. Breuer, M.A. Schmidt, "A wafer-bonded floating-element shear stress microsensor with optical position sensing by photodiodes," $J$. Microelectromechanical systems, Vol. 5, No. 4, pp. 307-15 (1996).

15. Y. Xu, F. Jiang, Q. Lin, J. Clendenen, S. Tung and Y.C. Tai, "Under water shear stress sensor," Fifteenth IEEE International Conference on Micro Electro Mechanical Systems (MEMS '02), Las Vegas, USA, (2002).

16. F.M. White, Fluid mechanics, $4^{\text {th }}$ Edition, McGraw-Hill (1999).

17. J. Ayers, P.M. Zavracky, N. McGruer, D. Massa, V. Vorus, R. Mukherjee, S. Currie, "A modular behavioral-based architecture for biomimetic autonomous underwater robots," Proc. of the Autonomous Vehicles in Mine Countermeasures Symposium. Naval Postgraduate School, CD ROM (1998); world-wide-web site http://www.cis.plym.ac.uk/cis/InsectRobotics/biomimetics. htm.

18. Y. Ozaki, T. Ohyama, T. Yasuda, I. Shimoyama, "An air flow sensor modeled on wind receptor hairs of insects", Proc. MEMS 2000, pp. 531-6, Miyazaki (2000).

19. Jun Zou, J. Chen and C. Liu, "Plastic deformation magnetic assembly (PDMA) of out-of-plane microstructures: Technology and application," IEEE/ASME Journal of $M E M S$, Vol. 10, No. 2, pp. 302-309 (2001).

20. W.G. Pfann and R.N. Thurston, "Semiconducting stress transducers utilizing the transverse and shear piezoresistance effects," J. Appl., Phys., Vol. 32, No. 10, pp. 2008-19 (1961).

21. S.V. Spoutai, "Practical model for electrical properties of highly doped p-type polysilicon," 4th International Conference on Actual Problems of Electronic Instrument Engineering Proceedings. APEIE-98, pp. 27-29, Novosibirsk, Russia (1998). 